\title{
Utilising Distributed Flexibilities in the European Transmission Grid
}

\author{
Manuel Ruppert, Viktor Slednev, Rafael Finck, Armin Ardone, \\ and Wolf Fichtner
}

\begin{abstract}
In this paper, we investigate the effect of distributed flexibilities on the operation of the transmission grid. The flexibilities considered are heat pumps, electric vehicles, battery energy storage systems and flexible renewable generation. For this purpose, we develop a two-stage approach of first determining an optimal electricity market solution considering the optimal dispatch of each generation element and flexibility. In the second step we determine the required dispatch adjustments due to transmission grid constraints and investigate the effect of integrating battery energy storage systems into the adjustable generators to solve congestions. In our case study, we investigate the central European transmission grid for a scenario based on the Distributed Generation scenario of the Ten-Year Network Development Plan for the year 2030. Integrating distributed flexibilities leads to a strong increase in the security of supply, while the overall effect on the generation adjustment is small. A comparison of the results for an AC and DC formulation shows that both approaches differ significantly in individual cases.
\end{abstract}

Keywords AC optimal power flow · Battery energy storage systems · DC optimal power flow · Distributed flexibilities · Distributed generation · Flexible demand · Transmission grid

\section{Introduction}

Today's change in the electricity system is driven by the decarbonisation initiative developed to meet emission reduction targets defined in international agreements in order to limit the global temperature increase. To reduce the carbon intensity of the electricity generation, an increasing amount of electricity generation from renewable sources (RES-E) is being installed throughout Europe. The two most

\footnotetext{
M. Ruppert $(\bowtie) \cdot$ V. Slednev $\cdot$ R. Finck $\cdot$ A. Ardone $\cdot$ W. Fichtner

Chair of Energy Economics, Institute for Industrial Production, Karlsruhe Institute of Technology (KIT), Karlsruhe, Germany

e-mail: manuel.ruppert@kit.edu
}

(C) The Author(s) 2020

V. Bertsch et al. (eds.), Advances in Energy System Optimization, Trends in Mathematics, https://doi.org/10.1007/978-3-030-32157-4_6 
significant sources are wind and solar, both characterised by volatility and their spatially distributed generation potential [1]. On the demand side, decarbonisation efforts have begun to lead to an increased electrification in the heat and transportation sectors. In some countries, rapid market growth of such elements which can be categorised as distributed flexibilities can be already be observed today. Future energy scenarios hint towards a further increase of these developments until 2030. With potentially high concurrencies of individual, decentralised demand patterns, uncontrolled or market-driven operation of these flexibilities will increase local as well as aggregated consumptions peaks in the power sector.

These changes pose significant challenges to the operation of the European transmission grid. First, balancing supply and demand will require additional flexibilities in a system dominated by distributed, intermittent RES-E and new sources of electrical demand. Second, RES-E at a specific point in time will be largely determined by the predominant meteorological conditions with high spatial concentration, increasing average distance between generation and consumption and thus increase the stress on the grid infrastructure. Lastly, market requirements to create a cost-minimal dispatch might contradict those necessary to enable a congestion-free transmission grid.

In the past, extensive research has been performed on different flexibility types concerning the technologies, their modelling and their contribution to renewable integration. A comprehensive overview on the current developments in electrical energy storage technologies and their potential for application in power system operation is given in [2] and [3]. Current reviews of different technological options can be found for power-to-heat applications [4], residential photovoltaic-battery energy storage (PV-BESS) [5], heat pumps (HP) in smart grids [6], and battery electric vehicles (BEV) [7]. Most literature focuses on the local balancing of demand and supply, either on a single household level or a community. This neglects the implications on the grid level or focuses on the regional effects in micro grids while including only a small set of technological options.

There are numerous models, which take into account flexibilities and simplified transmission grid constraints. In most cases, these models have been developed to analyse electricity markets, e.g. for investment decision support or operation decision support. In most studies liberalised markets in the United States or Europe are analysed and grid constraints are represented in a linearised way or by import/export constraints between market zones. Hence, most techno-economic models only take these constraints into account.

Few models, which consider flexibilities in a grid context take into account the full AC formulation of transmission grid constraints. Besides the group of studies and models, which consider a linearised form of grid constraints, some models can integrate full AC constraints. However, they are limited to either a very short time horizon or their focus lies on transient technical aspects like fault level detection, transient stability, harmonic analysis, reliability and power flow. Another group of models takes into account many flexibilities and AC restrictions but only on a distribution grid level, thus limiting system size and thereby complexity 
significantly [8]. A comprehensive overview on modelling approaches and their consideration of the grid can be found in [9].

Another area where transmission constraints are explicitly considered is the co-optimisation of transmission and generation capacities. The complexity of the resulting problem forces modellers to apply linearised transmission grid constraints or reduce the number of modelled flexibilities [10]. Furthermore, such models are often only validated on small scale or test grids, making it difficult to assess their suitability for large real-world power grids [11]. In most cases, the focus lies on investment decisions rather than on utilising operational flexibility in future transmission systems. [12] provide an overview of requirements and approaches to address this problem.

In this paper, we present a modelling framework to investigate the effect of distributed flexibilities on the transmission grid operation and investigate the benefit of utilising distributed flexibilities to reduce grid congestion while taking into account the full AC model of the transmission grid.

\section{Modelling the Interaction of Market and Grid Operation}

In order to model today's interaction between the electricity market and transmission grid operation, we developed a two-step approach to model market and grid operation. In the first step, we use a linear optimisation approach to determine the minimal-cost, copperplate-based results of the interconnected electricity market. In the second step, we determine the required dispatch adjustments due to congestion in the transmission grid using a multi-period nonlinear optimisation model. In the following section, the general modelling of the electricity market is described, followed by the integration of distributed flexibilities and the application of the method for modelling the transmission grid operation.

\subsection{Electricity Market}

To model the interconnected electricity market consisting of multiple market areas, we use a linear optimisation approach with the objective function of minimal total annual system cost under the assumptions of perfect foresight and perfect competition. ${ }^{1}$ The system cost consist of the aggregated variable cost of electricity

\footnotetext{
${ }^{1} \mathrm{~A}$ representation of the electricity market using an optimisation approach with cost minimisation objective function and perfect foresight does not necessarily allow for a realistic representation of market prices, as scarcitiy rents cannot be considered. As the market model is first and foremost utilised to obtain dispatch results for the transmission grid simulation in our application, using the described approach is a reasonable simplification for this purpose.
} 
production $C_{g}$ for the electricity generation $p_{g, t}$ of generator $\mathrm{g}$ in time step $\mathrm{t}$ and the cost of load shedding $C_{L S}$ for the amount of load shedding $p_{d, t}^{L S}$ of demand d in time step t.

$$
\min _{P_{g, t}, P_{d, t}} \sum_{g \in G, t \in T} C_{g} * p_{g, t}+\sum_{d \in D, t \in T} C_{L S} * p_{d, t}^{L S}
$$

In every market area $\mathrm{m}$, the electricity demand $P_{d, t}$ in every time step must be balanced out against generation, load shedding (LS) and interconnector flows into the market area $p_{c, t}$.

$$
\sum_{g \in G^{M}} p_{g, t}+\sum_{d \in D^{M}} p_{d, t}^{L S}+\sum_{c \in I C^{M}} p_{c, t}=\sum_{d \in D^{M}} P_{d, t} \forall m \in M, t \in T
$$

The bounds of each generator are determined by the minimum capacity $P_{g, t}^{\min }$ and maximum capacity $P_{g, t}^{\max }$ of the respective generating unit. While the boundaries of conventional generation are determined by the technical parameters of the generator, the boundaries of RES-E are determined by the available generation due to the intermittent nature. Available interconnection flows between market areas are restricted by the available exchange capacity $P_{c}^{\min }, P_{c}^{\max }$. In order to ensure feasibility of the problem, LS up to total demand can be performed at each time step.

$$
\begin{gathered}
P_{g, t}^{\min } \leq p_{g, t} \leq P_{g, t}^{\max } \forall g \in G, t \in T \\
P_{c}^{\min } \leq p_{c, t} \leq P_{c}^{\max } \forall c \in I C, t \in T \\
0 \leq p_{d, t}^{L S} \leq P_{d, t} \forall d \in D, t \in T
\end{gathered}
$$

\subsection{Modelling Flexibilities}

In the following, we apply a set of general equations to model various types of time-coupled flexibilities, ranging from different power storage technologies to consumption and renewable flexibilities. Ignoring self-discharging losses, the energy level $v_{s, t}$ of a storage $s \in S$ at time step $t \in T=\{1, \ldots N T\}$ equals its previous level, the external power inflow $\zeta_{s, t}^{\text {in }}$ or outflow $\zeta_{s, t}^{\text {out }}$ and the sum of charged and discharged power provided by connected generators $p_{g, t} \in G^{S} \subseteq G$ :

$$
\begin{gathered}
v_{s, t}=v_{s, t-1}+\sum_{g \in G^{i n S}} p_{g, t} * \eta_{g}-\sum_{g \in G^{\text {out } S}} p_{g, t} / \eta_{g}+\zeta_{s, t}^{\text {in }}-\zeta_{s, t}^{\text {out }} \\
\forall s \in S, t \in T
\end{gathered}
$$


For a simpler notation, the set of storage-connected generators $G^{S}$ is split such that $G^{\text {out } S}$ denotes those generators supplying power to the electricity system by discharging a storage and $G^{i n S}$ vice versa (charging of a storage). The charging and discharging efficiency is denoted by $\eta_{g}$. Furthermore, all variables might be restricted to lower and upper bounds and the starting condition for the storage is either a coupling of the first and last time steps or the definition of an initial storage level:

$$
\begin{gathered}
V_{s, t}^{\text {min }} \leq v_{s, t} \leq V_{s, t}^{\text {max }} \forall s \in S, t \in T \\
P_{g, t}^{\text {min }} \leq p_{g, t} \leq P_{g, t}^{\text {max }} \forall g \in G, t \in T \\
\mathrm{Z}_{t}^{\text {min }} \leq \zeta_{s, t} \leq Z_{t}^{\text {max }} \forall s \in S, t \in T \\
v_{s, o}=v_{s, N T} \vee v_{s, o}=V_{s}^{\text {start }} \forall s \in S
\end{gathered}
$$

\subsection{Storage Technologies}

Modelling the flexibility provided by actual storage technologies $S^{S} \subseteq S$ based on the equations shown above is straightforward and could easily be applied to hydro storages and batteries. Pure hydro pump storages and large-scale battery systems allow a rather simple definition of variable bounds. Storage volumes and generator capacities are non-negative and time independent, so that the equations for the energy level and power output can be reformulated.

$$
\begin{gathered}
0 \leq v_{s, t} \leq V_{s}^{\text {max }} \forall s \in S^{S}, t \in T \\
0 \leq p_{g, t} \leq P_{g}^{\text {max }} \forall g \in G^{\text {in } S^{s}}, t \in T \\
0 \leq p_{g, t} \leq P_{g}^{\text {max }} \forall g \in G^{\text {out } S^{s}}, t \in T
\end{gathered}
$$

While large-scale battery systems allow to ignore external power inflow and outflow, which do not result from indirectly connected generators or consumption, this is only true for non-cascading pump storage systems. However, we will ignore the case of cascading systems in the following and refer to the description of its modelling [13]. Including a time series of natural inflow and the restriction of a potential minimal outflow, allows for modelling mixed-hydro pump storages and/or pure seasonal storages, with an empty set $G^{i n S}$ in the last case. ${ }^{2}$

\footnotetext{
${ }^{2}$ In case of missing data for modelling seasonal storages, such as the actual volume or the inflow time series, it might be modelled as described in Sect. 2.5.
} 
Concerning small scale batteries, such as BESS combined with a PV system, the mathematical modelling is identical to large-scale battery systems, as long as no further restrictions apply to the battery utilisation. In practical application, however, regulators might apply specific restrictions for the operation of such systems. In Germany, for example, a widely used support scheme for PV-BESS is limiting the grid feed-in up to $50 \%$ of the PV capacity. ${ }^{3}$ Given a demand $P_{d, t}$ and a maximum grid feed-in $P_{g}^{l i m}$, these restriction might be easily implemented:

$$
\begin{gathered}
\sum_{g \in G^{P V^{s}}}\left(p_{g, t}-P_{g, t}^{l i m}\right)+\sum_{g \in G^{\text {out }} S^{s}} p_{g, t} \leq \sum_{d \in D^{s}} P_{d, t} \\
\forall s \in S^{P V B E S S}, t \in T
\end{gathered}
$$

\subsection{Demand Flexibilities}

In general, consumption processes with the ability to store energy can be directly modelled as single storages. Under the assumption that a certain share of a specific load might be shifted within a certain time range, the storage restrictions, however, might be applied to a broader range of demand flexibilities or for modelling the flexible potential of aggregated loads. Considering our target to model the impact of demand flexibilities at high and extra high voltage levels, we focus on modelling the flexibility for large-scale consumption process or of aggregated loads as storages $S^{L} \subseteq S$. When looking at the load shifting potential of an EV fleet, aggregation allows to neglect technical restrictions of single units and to derive the load shifting potential from the specific load profile [14]. This finding is supported by [15] where an aggregated modelling of BEV as flexible loads led to almost the same results in a unit commitment problem as their individual representation, after the BEVs where clustered accordingly.

Therefore, we model the demand flexibility of utility scale HP and the aggregation of numerous BEV and household heat pumps (HH-HP) by defining certain share $\alpha$ of a specific load $P_{d, t}$ as flexible $P_{d, t}^{\text {flex }}=\alpha^{\text {flex }} * P_{d, t}$ and adjust the general equations based on the load profile in a way such that $P_{d, t}^{\text {flex }}$ might be shifted within a specific time interval. Given a segmentation of the time interval $T=\left\{T T_{1}, \ldots, T T_{M}\right\}$ into $\mathrm{M}$ subsets, the storage equation might be reduced by

\footnotetext{
${ }^{3} \mathrm{KfW}-$ Kredit 275-Erneuerbare Energien-Speicher, from https://www.kfw.de/inlandsfoerderung/ Unternehmen/EnergieUmwelt/F\%C3\%B6rderprodukte/Erneuerbare-Energien-\%E2\%80\%93Speicher-(275)
} 
neglecting the variables for storage inflow and discharge:

$$
v_{s, t}=v_{s, t-1}+\sum_{g \in G^{i n S^{s}}} p_{g, t} * \eta_{g}-\zeta_{s, t}^{\text {out }} \forall s \in S^{L}, t \in T \backslash 1
$$

By restricting the storage outflow to the flexible demand profile

$$
\mathrm{Z}_{s, t}^{\text {min }} \leq \zeta_{s, t}^{\text {out }} \leq \mathrm{Z}_{s, t}^{\text {max }}=\sum_{d \in D^{s}} P_{d, t}^{\text {flex }} \forall s \in S^{L}, t \in T
$$

We define that the flexible demand either directly translates to a system load or is stored. In case that load shedding is not allowed, $\mathrm{Z}_{s, t}^{\min }$ equals $\mathrm{Z}_{s, t}^{\max }$. Assuming that the energy of a flexible demand profile has to be consumed within a time interval $T T_{m}$, the bounds of the storage volume are defined by the integral of the profile over $T T_{m}$ and equal zeros in the last time step.

$$
\begin{aligned}
& V_{s, t}^{\max }=-V_{s, t}^{\min }=\left\{\begin{array}{c}
0 \quad \text { if } t=\max \left\{T T_{m}\right\} \\
\sum_{t \in T T_{m}} \sum_{d \in D^{s}} P_{d, t}^{\text {flex }} \text { else }
\end{array}\right. \\
& \forall s \in S^{L}, m=1 \ldots M, t \in T
\end{aligned}
$$

Concerning the bounds for the storage discharge, the minimal or maximal values of the profile time series within the certain time interval $T T_{m}$ or the whole time horizon can be used.

$$
\begin{gathered}
P_{d, t}^{\max }=\min \left\{\max _{t \in T T_{m}} \alpha^{+} * P_{d, t}^{\text {flex }}, \max _{t \in T} P_{d, t}^{\text {flex }}\right\} \\
P_{d, t}^{\min }=\max \left\{\min _{t \in T T_{m}} \alpha^{-} * P_{d, t}^{\text {flex }}, 0\right\} \\
\forall d \in D^{s}, m=1 \ldots M, t \in T
\end{gathered}
$$

In order to model the specific flexible demand technologies, only the definition of the time intervals as well as $\alpha^{\text {flex }}, \alpha^{+}, \alpha^{-}$have to be adjusted, where $\alpha^{+}$and $\alpha^{-}$ are scaling parameters within the range of zero and infinity.

\subsection{Renewable Flexibilities}

Given a generation profile, a flexible operation of RES technologies, which depend on a storable resource, such as hydro- or bioenergy, might be modelled analogously to the case of flexible demand. This might be useful in cases where the actual storage parameters are unknown or for modelling virtual power plants, comprising multiple small units. The general idea is to model the flexibility by enabling a shift of the initial generation profile within a certain time interval. If a certain share $\alpha$ of the 
renewable generation is based on stored energy, we first split the generation variable into a flexible and inflexible part:

$$
\begin{gathered}
P_{g, t}^{\min } \leq p_{g, t}^{\text {inflex }}+p_{g, t}^{\text {flex }} \leq P_{g, t}^{\text {max }} \\
p_{g, t}^{\text {flex }} \leq \alpha^{\text {flex }} * P_{g, t}^{\text {max }}=P_{g, t}^{\text {flex }}
\end{gathered}
$$

Just like in the case of flexible demand, we assume that the flexible generation might be shifted within a specific time interval $T=\left\{T T_{1}, \ldots, T T_{M}\right\}$. In consequence, the flexible operation might be expressed by modifying the general storage equation:

$$
\begin{gathered}
v_{s, t}=v_{s, t-1}-\sum_{g \in G^{\text {out } S^{s}}} p_{g, t}^{\text {flex }} / \eta_{g}+\zeta_{s, t}^{\text {in }} \\
\forall s \in S^{R}, t \in T \backslash 1
\end{gathered}
$$

$S^{R}$ defines the set of storages used to model the generation shift flexibility of renewables. By restricting the storage inflow to the flexible generation profile

$$
\mathrm{Z}_{s, t}^{\text {min }} \leq \zeta_{s, t}^{i n} \leq \mathrm{Z}_{t}^{\text {max }}=\sum_{g \in G^{\text {out } S^{s}}} P_{g, t}^{\text {flex }}
$$

We define that the flexible renewable generation is either directly feed-in or stored. In case that dumping the flexible renewable generation is not allowed, $\mathrm{Z}_{s, t}^{\mathrm{min}}$ equals $\mathrm{Z}_{s, t}^{\max }$. Assuming that the energy of a flexible renewable generation profile has to be fed in within a time interval $T T_{m}$, the bounds of the storage volume are defined by the integral of the profile over $T T_{m}$ and equal zero in the last time step.

$$
\begin{aligned}
& V_{s, t}^{\max }=-V_{s, t}^{\min }=\left\{\begin{array}{c}
0 \quad \text { if } t=\max \left\{T T_{m}\right\} \\
\sum_{t \in T T_{m}} \sum_{g \in G^{\text {out } S^{s}}} P_{g, t}^{\text {flex }}
\end{array}\right. \text { else } \\
& \forall s \in S^{R}, m=1 \ldots M, t \in T
\end{aligned}
$$

Concerning the bounds for the storage discharge, the minimal or maximal values of the profile time series within the certain time interval $T T_{m}$ or the whole time horizon can be used.

$$
\begin{gathered}
P_{g, t}^{\max }=\min \left\{\max _{t \in T T_{m}} \alpha^{+} * P_{g, t}^{\text {flex }}, \max _{t \in T} P_{g, t}^{\text {flex }}\right\} \\
P_{g, t}^{\text {min }}=\max \left\{\min _{t \in T T_{m}} \alpha^{-} * P_{g, t}^{\text {flex }}, 0\right\} \\
\forall g \in G^{\text {out } S^{s}}, t \in T
\end{gathered}
$$


In order to model the specific flexible renewable technologies, only the definition of the time intervals and $\alpha^{\text {flex }}, \alpha^{+}, \alpha^{-}$has to be adjusted.

\subsection{Grid Operation}

Based on the dispatch result for each generation unit and time step from the market model, we determine the necessary dispatch adjustment due to grid constraints in the second step using a nonlinear optimisation approach. As a conventional cost minimising objective function under consideration of grid constraints would lead to a nodal pricing result, which would discard the dispatch determined by the market, we establish the minimum amount of dispatch adjustment in the entire network as the optimisation objective and evaluation figure to assess the efficiency in the transmission grid. Therefore, the generalised objective function can be formulated as follows:

$$
\min _{\Delta p_{g, t}, \Delta p_{d, t}^{L S}} \sum_{g \in G, t \in T}\left|\Delta p_{g, t}\right|+\sum_{d \in D} \Delta p_{d, t}^{L S}
$$

Positive and negative deviation of generation units from the base solution $\Delta P_{g, t}$ are considered equivalently towards the objective, while grid-induced load shedding $\Delta P_{d, t}^{L S}$ is unidirectional by definition.

We solve the resulting optimisation problem with a multi-period nonlinear formulation of the ACOPF utilising an augmented Lagrangian formulation for coupling of time-dependent storage units as described in [16]. In order to eliminate the possibility of results representing significantly suboptimal solutions caused by converging into local optima due to the nonlinear nature of the problem, we benchmark our results with a DCOPF formulation, as described in [17].

\section{Case Study}

In the following, we apply the presented modelling approach on a scenario derived from the scenario "Distributed Generation" (DG) of the European Network of Transmission System Operators for Electricity (ENTSO-E) Ten-Year Network Development Plan (TYNDP) 2016. In our study, we restrict the scope of both electricity market and grid to the central European countries and their transmission networks, thus not considering the effect of flows from further interconnected countries. The considered area contains the countries France, Belgium, Netherlands, Switzerland, Austria, Czech Republic, Poland, Germany and Luxembourg. These countries form individual market areas, with the exception of Germany and Luxembourg, which form a common market area. 
In addition to the scenario data from the DG scenario, the closely related high renewables scenario $\mathrm{C}$ of the german network development plan 2030 was used for Germany due to the fact that this source includes data with a higher level of detail. Based on the projected national RES capacity development of the scenario an optimal allocation planning model as described in [18] was run in the first step for regionalisation. In detail, a cost optimal expansion planning with a high spatial resolution until 2050 is performed, considering national and regional lower and upper bounds for the development of single renewable technologies. Due to the longer optimization horizon, exceeding the forecast horizon of the underlying scenarios, a minimal RES-E share on the demand is defined in the later years, such that an $80 \%$ renewable share is achieved in Europe until 2050.

In the second step, the capacity adequacy is analysed based on a MonteCarlo simulation of the generation availability. Based on an integrated European approach, accounting for a cross-border balancing, and the availability of a flexible demand shift (BEV and heap pumps in households and district heating) and flexible renewables (hydro storage and biomass) we computed the needed dispatchable capacity. ${ }^{4}$ Afterwards we utilised results obtained from a generation expansion planning problem of the European power system in order to meet the calculated capacity demand for a secure operation of the system [19].

In the current scenario, we assumed that existing power plants are decommissioned at the end of their technical lifetime. For coal-fired power plants, a premature decommissioning pathway until 2040 is assumed and individually adjusted for each unit with regard to its technical parameters and national regulations, aligned as much as possible with the political guidelines on coal phase out known today. The portfolio of hydro power plants with storage or pump-storage capability is based on today's generation and announced construction or expansion projects under the assumption, that generators reaching the end of their lifetime are replaced with the same parameters.

In the presented case study, 555 existing or currently projected and 145 new built thermal power plants and 110 hydro power plants are operational in the scenario in 2030. The resulting thermal, hydro pump and hydro turbine capacity and the location of their connection to the transmission grid are shown in Fig. 1.

The case study year with input data for transmission grid as well as generation and demand is chosen as 2030 and the simulations are performed with an hourly time granularity. For the transmission grid part, a weekly optimization horizon of transmission grid without rolling horizon was chosen. For this, storage states of each unit at start and end time were fixed to the state given by the previously determined state after market-based dispatch.

\footnotetext{
${ }^{4}$ The results showed a mismatch between the calculated adequate capacity and the capacity in the scenario, which was significantly lower in some countries. The missing DSM modelling might explain some part of the mismatch.
} 


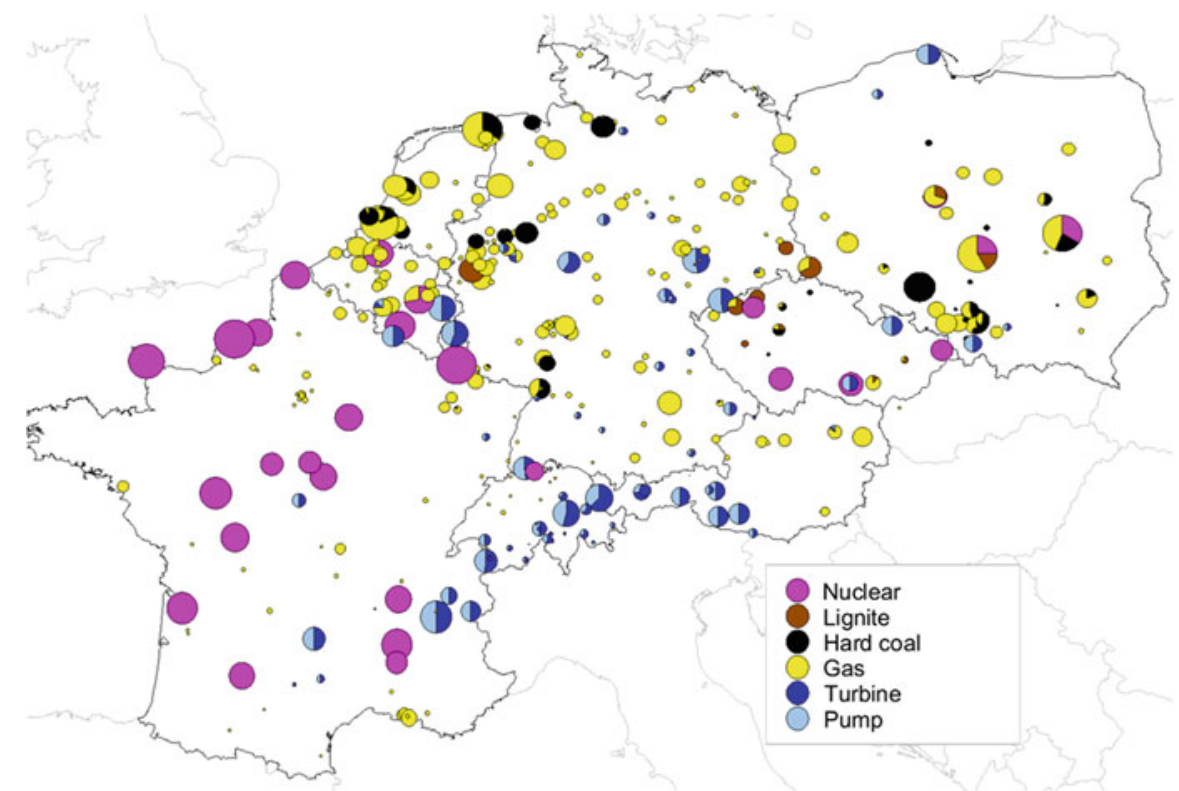

Fig. 1 Installed capacity per fuel type at the transmission grid substations. The circle volume is proportional of the legends circle volume of $2 \mathrm{GW}$

\subsection{Transmission Grid Model}

The transmission grid model contains the interconnected AC network of the same countries considered on the market side, including overhead lines and cables of voltage levels above $200 \mathrm{kV}$. For the studied area, this includes the nominal voltage levels of 220 and $380 \mathrm{kV}$. Additionally, high voltage DC (HVDC) lines are included in the dataset. HVDC lines connecting offshore wind generation are considered at the point of RES-E calculation and allocation and subsequently reduced from the dataset. All AC and HVDC lines are connected to busbars, which are assigned to georeferenced substations. The number of busbars at each substation is limited to the number of voltage levels present at the respective substation. In addition to the transformation between the extra high voltage levels, the transformation to the high voltage levels between 60 and $150 \mathrm{kV}$ is considered and used for the connection of small-scale generation and demand as described in [18].

The data include the present state of the transmission grid as well as projected expansion measures in terms of deconstruction, replacement and construction of substations, busbars, lines and transformers until the year 2030. The technical data for each grid element was derived from publicly available sources whenever possible and otherwise approximated based on available information from predominant comparable equipment in the same geographical and organisational area. Among the sources used for completing the dataset are the static grid models of the transmission 
grid operators of the Central Western Europe Region (CWE), as well as the grid dataset of the TYNDP 2016 and Open Street Map (OSM). The future expansion of the national grid networks was obtained from various sources such as national network development plans of the respective grid operators and the projects listed in the TYNDP 2016. The model represents a snapshot of the projected grid topology in 2030 and includes a total of 3010 busbars, distributed over 1513 substations. The busbars are connected by 4103 AC lines and transformers, as well as six HVDC lines, with the exception of the interconnector between Belgium and Germany being located in the German market area. Furthermore, 237 reactive power compensation elements are located in the entire system, with either positive or negative reactive power provision. The snapshot of the transmission grid topology for 2030 is shown in Fig. 2.

The interconnection capacities between market areas are determined based on the available thermal capacities of interconnecting lines from the transmission grid dataset, assuming that $40 \%$ of the total thermal capacity of interconnecting AC lines between the market areas and the full thermal capacity of interconnection HVDC lines is being made available for market operation. Generators from nonintermittent sources are assumed to have reactive power provision capabilities of $\cos \varphi=0.925$ w.r.t the installed generator capacity. Furthermore, RES-E with a nodal distribution of installed capacity as shown in Fig. 3 is assumed to be able to

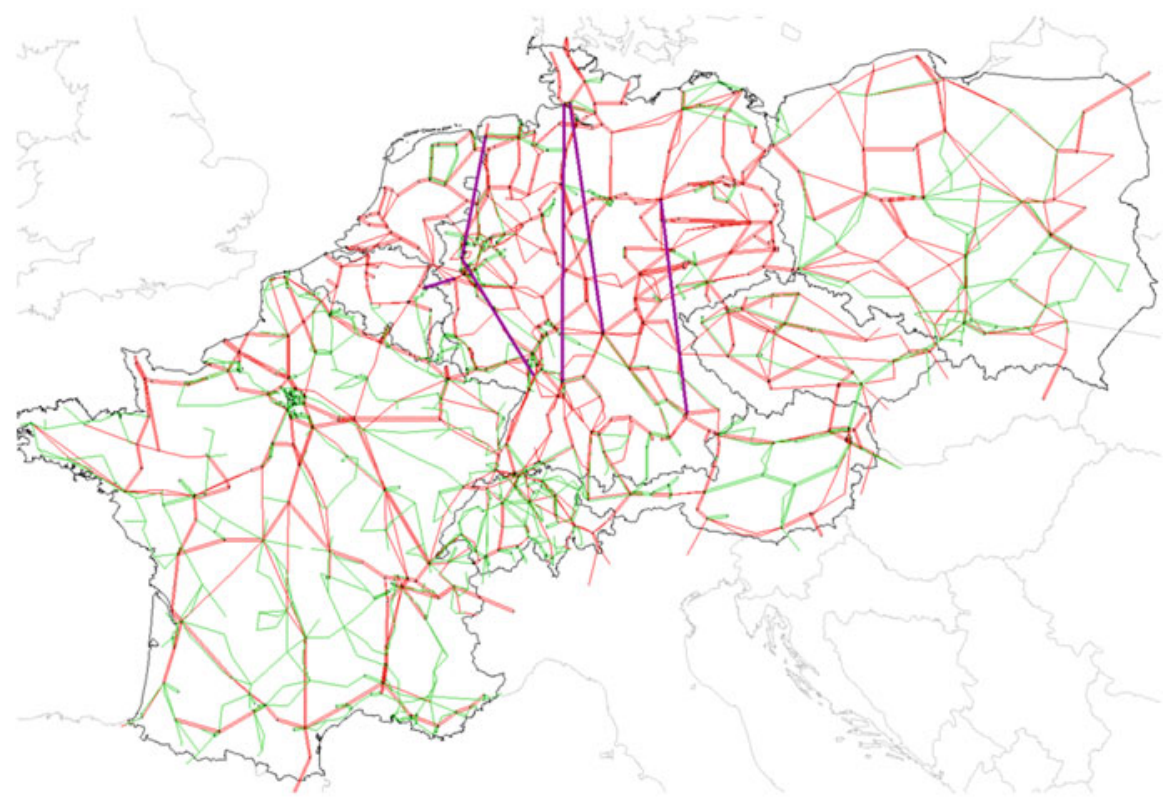

Fig. 2 Map of the transmission 2030 used in the case study. Red denotes $380 \mathrm{kV}$ lines, green $220 \mathrm{kV}$ lines and purple HVDC lines 


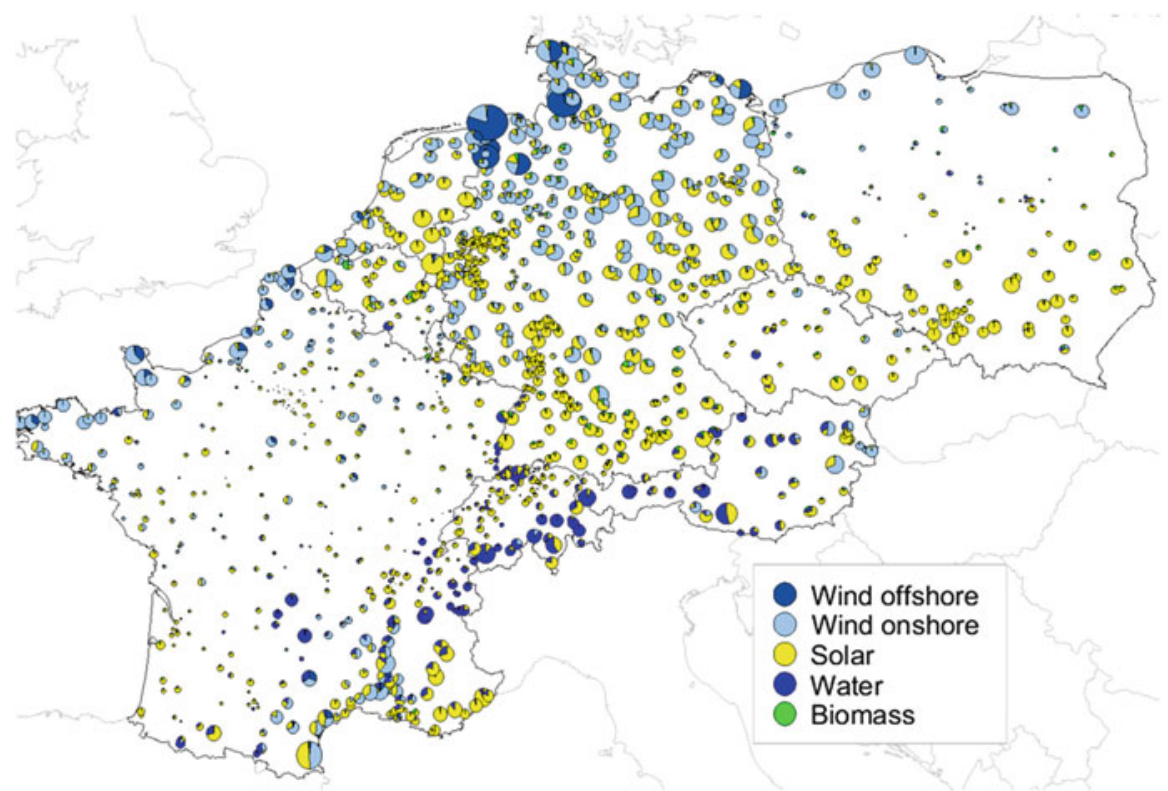

Fig. 3 Installed renewable capacity per type at the transmission grid substations. The circle volume is proportional to the legends circle volume of $2 \mathrm{GW}$

provide reactive power up to a level of $\cos \varphi=0.95$ for all sources of renewable energy. With regard to the reactive power consumption, the reactive power demand in every market area and of every demand type is assumed to remain constant over time with a lagging power factor of $\cos \varphi=0.95$. The maximum voltage deviation from nominal voltage is restricted to $\pm 10 \%$ and grid components can be utilised up to their continuous thermal current restriction.

\subsection{Parameters for Flexibility Modelling}

Considering the modelling of new consumers, their flexibility and their regionalisation the following assumptions were made:

\subsubsection{Heat Pumps in Households}

The modelling of electricity consumed due to heat demand in households from HPs follows the approach of the Munich City Utilities (SMW) for calculating the standard load profile of HPs within their grid, based on the yearly household load and the temperature. Assuming a full flexibility $\left(\alpha^{\text {flex }}=1, \alpha^{+}=\right.$ $\left.1, \alpha^{-}=0\right)$ within a $3 \mathrm{~h}$ horizon $([00: 00-03: 00),[03: 00-06: 00), \ldots)$, 
the regionalisation follows the same approach as that of the household demand, restricted to single and two family buildings.

\subsubsection{Heat Pumps in District Heating}

We apply the same approach for modelling the generation profile and its flexibility as it is the case for HH-HP. Due to the availability of a heating grid and the possibility to store larger amounts of energy, a full flexibility within a $24 \mathrm{~h}$ horizon $[00: 00-24: 00)$ is assumed. The regionalisation is in general based on the capacity of combined heat and power (CHP) units within a country. In Germany furthermore the data of the German District Heating Association (AGFW) for the actual district heating grids demand and location where combined with the CHP database for a more detailed analysis.

\subsubsection{BEV PBEV}

Profiles are based on $[14,20]$ for a direct charging at arrival and adjusted based on Table 1.

The regionalisation corresponds to that of the household demand in all European countries except of Germany, where the vehicle registration numbers at NUTS 3 level were used as a distribution key instead of household number and electricity demand. Concerning the flexibility, we assumed the potential to shift the full demand $\left(\alpha^{\text {flex }}=1, \alpha^{+}=1, \alpha^{-}=0\right.$ ) within a $12 \mathrm{~h}$ horizon either during the day or the night $([06: 00-18: 00),[18: 00:-06: 00)$.

\subsubsection{PV-BESS}

In the current analysis, decentral BESS installed with PV on household level are modelled as simple battery storages and differ only in their sizing and regionalization from classical battery storages. Based on a linear relation between household demand and self-optimised PV-BESS capacity, obtained from a mixed integer optimization of PV-BESS sizing of households [21] in northern Germany and

Table 1 BEV parameter set

\begin{tabular}{l|l|l|l|l|l}
\hline & $\begin{array}{l}\text { Charging at } \\
\text { home }[\mathrm{kW}]\end{array}$ & $\begin{array}{l}\text { Charging at } \\
\text { work }[\mathrm{kW}]\end{array}$ & Range $[\mathrm{km}]$ & $\begin{array}{l}\text { Mean distance } \\
{[\mathrm{km} / \mathrm{a}]}\end{array}$ & $\begin{array}{l}\text { Demand } \\
{[\mathrm{kWh} / 100 \mathrm{~km}]}\end{array}$ \\
\hline 2020 & 3,5 & 0 & 200 & 13.073 & 22 \\
\hline 2030 & 5 & 5 & 300 & 13.071 & 22 \\
\hline 2040 & 7 & 7 & 400 & 13.364 & 22 \\
\hline 2050 & 11 & 11 & 400 & 13.481 & 22 \\
\hline
\end{tabular}


southern Germany, an upper bound for the regional distribution of PV-BESS is calculated. By adjusting the BESS capacity according to the actual installed rooftop PV smaller $15 \mathrm{kWp}$ in single and two family buildings, the key for the final PV-BESS distribution is computed.

\subsubsection{RES-E Flexibilities}

For the flexibility of hydro storage and biomass generation we assumed $\alpha^{\text {flex }}=$ $0.5, \alpha^{+}=\inf , \alpha^{-}=0$, meaning that half of the generation profile is assumed to be flexible and restricted to the maximum value of the total time series of the flexible part. Furthermore, the potential to shift the generation within $24 \mathrm{~h}$ was assumed in case of biomass and of $168 \mathrm{~h}$ in case of hydro storage.

\section{Results}

\subsection{Scenarios}

In the following, we will present the results obtained from the simulations performed with the input dataset described above. The results are presented for four scenarios, which differ due to the amount of utilisation of the flexibilities both on the market and the transmission grid side. In this, we divide the available flexibilities into traditional flexible generation from hydro storage and into distributed flexibilities (HP, BEV, BESS, RES-E flexibilities) In the Base scenario, distributed flexibilities remain static according to their inelastic profile and time-dependent generation is not available for adjustment during grid operation. Thus, only hydro flexibilities are being utilised in the market operation and are subsequently fixed during the second step of grid operation simulation. The NoFlex scenario extends the Base scenario by adding hydro flexibilities to the available measures while resolving transmission grid congestion, keeping distributed flexibilities inelastic. The Flex scenario represents a further flexibilisation, with distributed flexibilities included in the market operation as described in Sects. 2.2, 2.3, 2.4, and 2.5 Lastly, in the DynFlex scenario the available BESS capacities after the market dispatch are added to the available adjustments during grid operation, increasing the number of storage units considered in the grid operation by 896 nodal distributed elements.

\subsection{The Impact of Distributed Flexibilities}

The yearly aggregated results are shown in Fig. 4. Transmission lines which reach their continuous thermal current limit during the time horizon are colored 


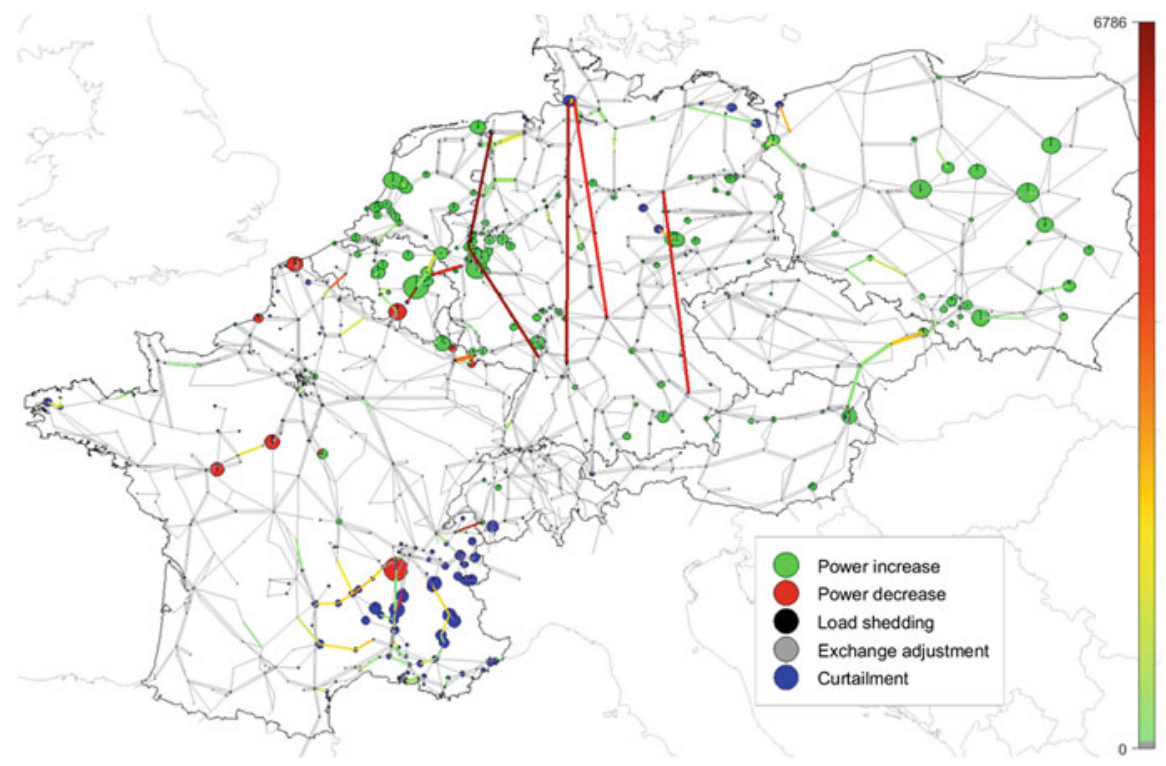

Fig. 4 Hours with maximum loading of transmission grid lines and average dispatch adjustment per type. The circle volume is proportional to the legends circle volume of $500 \mathrm{MW}$

according to the number of hours where this occurs, marking overloaded lines which require adjustments in the generation and demand dispatch in order to resolve the congestion. Congestions on the transformation level and voltage boundaries are omitted from Fig. 4. The highest number of fully loaded hours is found on the HVDC lines, implying that the start and end locations of these lines are generally well suited for relieving the stress on the AC grid. In the AC grid, areas with significant congestion can be found in Southern France, the border of France and Belgium, Northern Germany and the border area of Poland, the Czech Republic and Austria. Generally, the required decrease in generation at certain locations is reached by renewable curtailment rather than power decrease of thermal power plants. A trend of required power reduction in the southern part of the network can be observed over the entire year, while power increase is more focussed in the northern and eastern areas. When interpreting the results, possible discrepancies between the generation and demand scenario on the one hand and the grid model on the other hand have to be considered. For example, the grid expansion considered based on the TYNDP in France is significantly lower than the evolution of the grid in Germany in the time frame between today and 2030. Thus, the increase of both RES-E and new electricity demand types has an higher impact on a system that does not undergo a significant transformation.

The impact of the utilisation of distributed flexibilities on the security of supply can be seen in the reduction of the required load shedding in Table 2. While both the Base and the NoFlex scenario require load shedding in order to achieve a feasible 
Table 2 Load shedding results

\begin{tabular}{l|l|l|l|l}
\hline & Base & NoFlex & Flex & DynFlex \\
\hline Hours with LS & 240 & 136 & 11 & 9 \\
\hline Avg. hourly LS [MW] & 20,48 & 9,00 & 0,78 & 0,78 \\
\hline Max. hourly LS [MW] & 10043 & 6429,48 & 1794,14 & 2156,31 \\
\hline
\end{tabular}

solution in more than 100 single hours during the year, this value is greatly decreased in the Flex scenario and even more in the DynFlex scenario. The main reason for this is the reduction in peak demand and generation due to the market-based dispatch of flexibilities as described in Sect. 2. Furthermore, the additional control capabilities - albeit with a small capacity over a longer time horizon - lead to less congestion events, which cannot be prevented without curtailing demand.

In the Base scenario, the average hourly positive dispatch adjustment requirement in the entire transmission grid area considered is $8.672 \mathrm{GW}$, with peaks reaching adjustments of up to $18.5 \mathrm{GW}$. As a permanent additional power provision is required in order to balance the transmission losses over lines and transformers, the positive adjustment does not decrease to zero even in cases without any violation of voltage or thermal limits. In the NoFlex scenario, the hourly requirement reduces by $48 \mathrm{MW}$ while the peak increases to $20.1 \mathrm{GW}$. The hourly average decreases further to $8.54 \mathrm{GW}$ in the Flex scenario and $8.497 \mathrm{GW}$ in the DynFlex scenario, with the peak decreasing to 17.7 and $17.6 \mathrm{GW}$. The average negative dispatch adjustment required is not distorted by additional grid losses, over the scenarios it decreases by $3.6 \%, 2.1 \%$ and $1.0 \%$ from the NoFlex to the DynFlex scenario. Similarly to the positive adjustments, the negative yearly peak values are reaching the highest absolute values for the NoFlex scenario and the lowest for the DynFlex scenario.

Overall, the results show that as expected more flexibilities available both in the market and transmission grid lead to a lower amount of required redispatch. Yet, the different utilisations of both central and distributed flexibilities have a lower impact on the grid operation than expected. Among flexibilities the inclusion of largescale hydro in the Flex scenario has the largest impact, as observed in the yearly values of negative dispatch adjustment. Operating distributed flexibilities according to central market signals leads to a small improvement in terms of transmission grid congestion. A similar observation can be made for the inclusion of BESS in the adjustable generation on the grid side. The comparably small difference between the scenarios might be explained by the discrepancies in the transmission grid model and the generation and demand data for 2030, with structural congestion accounting for a large part of the observed overloading events. However, the resulting security of supply is largely increased by utilising distributed flexibilities with load shedding mostly rendered unnecessary, as can be seen in Table 2. In order to improve the accuracy of findings for the total amount, more grid expansion in the input data or a better coordination of renewable expansion and demand increase on the one hand and the transmission grid on the other hand might be needed. 


\subsection{AC vs. DC Formulation}

The comparison of hourly results of the DynFlex scenario with the results of an identical simulation performed with the DC restrictions in the load flow equations is shown in Fig. 5. For the sake of an equivalent comparison, grid losses are subtracted from the positive dispatch adjustment of the AC solution, and the demand of the DC model was increased by today's average national transmission grid losses for all market areas considered. Otherwise, both model formulations, restrictions and input data were kept consistent. The DC problem was solved using the commercial solver Gurobi. Even though the set of DC constraints form a relaxed formulation of the problem, the $\mathrm{AC}$ solution has a lower requirement for adjustments after correcting the AC results for the required adjustments to account for grid losses. This is due to the additional positive generation increase needed to account for grid losses in the AC case, which are part of the optimisation problem in the formulation chosen in this paper and thus contribute to relieving existing congestions. As a result they lower the additionally needed adjustment after the subtraction of the losses, leading to lower requirements, even though the linearised DC formulation is commonly referred to as a relaxation of the $\mathrm{AC}$ formulation. In the section up to $5 \mathrm{GW}$, the effect of voltage limits can be seen, which leads to higher AC adjustments as voltage is constant in the DC formulation. Overall, individual deviation in single hours can be significant while the entire trend and structure are similar. The correlation between the positive adjustment value series is $91.1 \%$. This shows that the DC approach is suitable as an estimator for the AC equations for the investigation of general trends, while individual results and peaks might differ significantly due to the simplifications undertaken. For these cases, the AC formulation leads to better results.
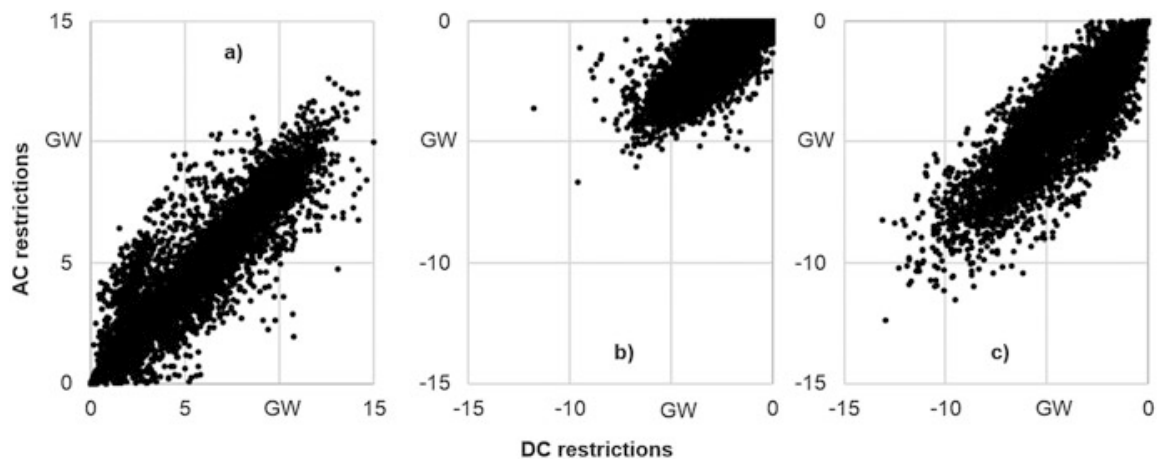

Fig. 5 (a) Distribution of positive adjustments for AC/DC results. (b) Distribution of negative adjustments of non-intermittent generators. (c) Distribution of negative adjustments of intermittent generators 


\section{Conclusion}

In this paper, we presented a two-step approach to investigate the influence of distributed flexibilities on the operation of the transmission grid in the central European area. For this, we have developed a market optimisation formulation, which enables us to obtain dispatch results which include different kind of distributed flexible generation and demand sources. Subsequently, we analysed the effect of four scenarios with different implementation rates of flexibilities in market and grid operation based on a case study using data for the year 2030. Furthermore, we compared the results of our multi-period $\mathrm{AC}$ formulation to determine grid congestion with a linearised DC formulation.

We conclude based on the required adjustments over the simulation horizon of $8760 \mathrm{~h}$, that a scenario like the DG scenario of the TYNDP, with high RES$\mathrm{E}$ increase and the additional introduction of demand-side flexibilities into the electricity system is generally feasible for the anticipated transmission grid of the year 2030. Locally concentrated, larger adjustments can be explained by the gap between known grid expansion in selected countries and the ambitious targets of the chosen scenario. Also, distributed flexibilities show to have a strong effect on solving grid congestions that lead to load shedding, which is necessary in the Base scenario of our study in more than $200 \mathrm{~h}$ in order to achieve a feasible solution. This is almost entirely resolved when optimising distributed flexibilities with the market and enabling the adjustment of BESS during grid operation.

Furthermore, the results of the four scenarios investigated show that the impact of increased participation of both central and distributed flexibilities in the market and grid operation has a positive but comparably low influence on the overall required adjustment to operate the grid within its technical limits in our chosen case study. The largest change on the average adjustments required occurs when adding central storage units to the set of generators and consumers available for adjustment in the grid simulation. The comparison of the AC and DC results showed that the required adjustment can be lower in the AC case, if the provision of grid losses is included into the AC formulation and generation increase is performed at locations which are suitable for lowering stress on the grid. Additionally, the individual results of each hour showed a generally well reproduced trend using the DC relaxation, however single hours can differentiate significantly in both cases.

In the current state a simplified approach of modelling the flexible operation of shiftable demands and renewables based on their profile was integrated in the market model. In the future we are planning to integrate the decentral flexibilities modelling into the grid model und validate it against a more detailed representation of individual units. As demand and supply uncertainty have a major impact on the utilisation of storages and load shifting potentials, we are furthermore planning to extend our modelling to a stochastic optimization. Finally, the extension of the current modelling approach to a unit commitment problem would allow us to fully evaluate the contribution of flexibilities for the future power system. 
Acknowledgements Manuel Ruppert and Rafael Finck gratefully acknowledge funding by the German Federal Ministry of Education and Research (BMBF) within the Kopernikus Project ENSURE "New ENergy grid StructURes for the German Energiewende".

Viktor Slednev kindly acknowledges the support for this work from the German Research Foundation (DFG) under the Project Number LE1432/14-2.

\section{References}

1. D. Keles, P. Jochem, R. McKenna, M. Ruppert, W. Fichtner, Meeting the modeling needs of future energy systems. Energy Technol. (2017). https://doi.org/10.1002/ente.201600607

2. X. Luo, J. Wang, M. Dooner, J. Clarke, Overview of current development in electrical energy storage technologies and the application potential in power system operation. Appl. Energy (2015). https://doi.org/10.1016/j.apenergy.2014.09.081

3. M. Beaudin, H. Zareipour, A. Schellenberglabe, W. Rosehart, Energy storage for mitigating the variability of renewable electricity sources: an updated review. Energy Sustain. Dev. (2010). https://doi.org/10.1016/j.esd.2010.09.007

4. A. Bloess, W.-P. Schill, A. Zerrahn, Power-to-heat for renewable energy integration: a review of technologies, modeling approaches, and flexibility potentials. Appl. Energy 212, 1611-1626 (2018)

5. J. Hoppmann, J. Volland, T. Schmidt, V. Hoffmann, The economic viability of battery storage for residential solar photovoltaic systems - a review and a simulation model. Renew. Sustain. Energy Rev. (2014). https://doi.org/10.1016/j.rser.2014.07.068

6. D. Fischer, H. Madani, On heat pumps in smart grids: a review. Renew. Sustain. Energy Rev. (2017). https://doi.org/10.1016/j.rser.2016.11.182

7. D. Richardson, Electric vehicles and the electric grid: a review of modeling approaches, impacts, and renewable energy integration. Renew. Sustain. Energy Rev. (2013). https://doi. org/10.1016/j.rser.2012.11.042

8. D.P. Chassin, K. Schneider, C. Gerkensmeyer, GridLAB-D: an open-source power systems modeling and simulation environment, in Exposition, Chicago, pp. 1-5. https://doi.org/10. 1109/TDC.2008.4517260

9. H.-K. Ringkjøb, P. Haugan, I. Solbrekke, A review of modelling tools for energy and electricity systems with large shares of variable renewables. Renew. Sust. Energ. Rev. (2018). https://doi. org/10.1016/j.rser.2018.08.002

10. C. de Jonghe, B.F. Hobbs, R. Belmans, Optimal generation mix with short-term demand response and wind penetration. IEEE Trans. Power Syst. (2012). https://doi.org/10.1109/ TPWRS.2011.2174257

11. L. Baringo, A.J. Conejo, Transmission and wind power investment. IEEE Trans. Power Syst. (2012). https://doi.org/10.1109/TPWRS.2011.2170441

12. V. Krishnan, J. Ho, B.F. Hobbs, A.L. Liu, J.D. McCalley, M. Shahidehpour, Q.P. Zheng, Co-optimization of electricity transmission and generation resources for planning and policy analysis: review of concepts and modeling approaches. Energy Syst. (2016). https://doi.org/10. 1007/s12667-015-0158-4

13. M. Ruppert, V. Slednev, V. Bertsch, W. Fichtner, The impact of microeconomic decisions in electricity market modelling on load flows in transmission grids, in 2016 13th International Conference on the European Energy Market (EEM). 2016 13th International Conference on the European Energy Market (EEM), 2016

14. P. Jochem, T. Kaschub, A.-G. Paetz, W. Fichtner, Integrating electric vehicles into the German electricity grid - an interdisciplinary analysis. World Electric Veh. J. (2012). https://doi.org/10. 3390/wevj5030763

15. D. Madzharov, E. Delarue, W. D'haeseleer, Integrating electric vehicles as flexible load in unit commitment modeling. Energy (2014). https://doi.org/10.1016/j.energy.2013.12.009 
16. M. Ruppert, V. Slednev, A. Ardone, W. Fichtner, Dynamic optimal power flow with storage restrictions using augmented lagrangian algorithm, in 2018 Power Systems Computation Conference, Dublin (IEEE, 2018). https://doi.org/10.23919/PSCC.2018.8442993

17. S. Frank, S. Rebennack, An introduction to optimal power flow. Theory, formulation, and examples. IIE Trans. (2016). https://doi.org/10.1080/0740817X.2016.1189626

18. V. Slednev, M. Ruppert, V. Bertsch, W. Fichtner, N. Meyer-Hübner, M. Suriyah, T. Leibfried, P. Gerstner, M. Schick, V. Heuveline, Regionalizing input data for generation and transmission expansion planning models, in Advances in Energy System Optimization, ed. by V. Bertsch, W. Fichtner, V. Heuveline, T. Leibfried. Trends in Mathematics (Springer International Publishing, Cham, 2017), pp. 205-217

19. R. Hartel, V. Slednev, U. Yilmaz, A. Ardone, D. Keles, W. Fichtner, A. Eser, M. Klobasa, M. Kuhnbach, P. Manz, J. Globisch, R. Elsland, M. Wietschel, Dekarbonisierung des Energiesystems durch verstärkten Einsatz erneuerbaren Stroms im Wärme-, Verkehrs-und Industriesektor bei gleichzeitigen Stilllegungen von Kraftwerken - Auswirkungen auf die Versorgungssicherheit in Süddeutschland (2018)

20. V. Slednev, V. Bertsch, M. Ruppert, W. Fichtner, Highly resolved optimal renewable allocation planning in power systems under consideration of dynamic grid topology. Comput. Oper. Res. (2018). https://doi.org/10.1016/j.cor.2017.12.008

21. T. Kaschub, P. Jochem, W. Fichtner, Solar energy storage in German households: profitability, load changes and flexibility. Energy Policy (2016). https://doi.org/10.1016/j.enpol.2016.09.017

Open Access This chapter is licensed under the terms of the Creative Commons Attribution 4.0 International License (http://creativecommons.org/licenses/by/4.0/), which permits use, sharing, adaptation, distribution and reproduction in any medium or format, as long as you give appropriate credit to the original author(s) and the source, provide a link to the Creative Commons licence and indicate if changes were made.

The images or other third party material in this chapter are included in the chapter's Creative Commons licence, unless indicated otherwise in a credit line to the material. If material is not included in the chapter's Creative Commons licence and your intended use is not permitted by statutory regulation or exceeds the permitted use, you will need to obtain permission directly from the copyright holder. 